\title{
Impaired lung function is associated with non-alcoholic fatty liver disease independently of metabolic syndrome features in middle-aged and elderly Chinese
}

\author{
Li Qin ${ }^{1,2+}$, Weiwei Zhang ${ }^{1,2+}$, Zhen Yang ${ }^{1,2^{*}}$, Yixin Niu ${ }^{1,2}$, Xiaoyong Li ${ }^{1,2}$, Shuai Lu' ${ }^{1}$, Yin Xing ${ }^{1}$, Ning Lin ${ }^{1,2}$, \\ Hongmei Zhang ${ }^{1,2}$, Guang Ning ${ }^{3}$, Jiangao Fan ${ }^{4}$ and Qing Su ${ }^{1,2^{*}}$
}

\begin{abstract}
Background: Associations between lung function and non-alcoholic fatty liver disease (NAFLD) have been reported. However, evidence from large-scale populations about the relationship is scarce. The objective of the study was to evaluate the relationship between lung function and NAFLD in middle-aged and elderly Chinese.

Methods: A total of 1842 participants aged 40 years or older were recruited from Chongming District, Shanghai, China. Lung function, evaluated by forced vital capacity (FVC) and forced expiratory volume in one second (FEV1) was measured with standard spirometry. The NAFLD was evaluated by ultrasonography.
\end{abstract}

Results: The subjects with NAFLD had lower FVC (\% predicted) $(0.85 \pm 0.26$ vs. $0.90 \pm 0.28, p<0.001)$ and FEV1 (\% predicted) $(0.93 \pm 0.29$ vs. $0.98 \pm 0.34, p<0.001)$ than non-NAFLD. After adjusting for potential risk factors, the lowest quartile of FVC (\% predicted) and FEV1 (\% predicted) was associated with increased prevalence of NAFLD, with the fully adjusted odds ratio of 1.37 and 1.24 (95\% confidence interval [Cl] 1.18-1.97, $p<0.001,95 \% \mathrm{Cl} 1.11-1.87$, $p=0.009)$, respectively.

Conclusions: Impaired lung function is associated with non-alcoholic fatty liver disease, independent of conventional metabolic risk factors.

Keywords: Lung function, Non-alcoholic fatty liver disease, Chinese, Metabolic risk factors

\section{Background}

Nonalcoholic fatty liver disease (NAFLD) is characterized by excessive hepatic fat accumulation of patients who have no history of alcohol abuse [1]. Recently, the combination of overnutrition condition and less physical activity have made NAFLD become the most common disease of chronic liver damage, with increased prevalence of obesity, diabetes, and metabolic syndrome in developed and developing counties [2]. The traditional

\footnotetext{
* Correspondence: yangzhen1020@hotmail.com; suqingxinhua@163.com ${ }^{\dagger}$ Equal contributors

${ }^{1}$ Department of Endocrinology, Xinhua Hospital Chongming Branch, Shanghai Jiao Tong University School of Medicine, Shanghai, China

Full list of author information is available at the end of the article
}

risk factors of NAFLD, such as central obesity, insulin resistance, systemic inflammation, current smoking, diabetes, and oxidative stress, contribute to, but cannot fully explain the increased risk of NAFLD in the general population [3-5].

Recently, lung function parameters, estimated by forced vital capacity (FVC) and forced expiratory volume in one second (FEV1) were proved to be well associated with the development of diabetes, cardiovascular disease, inflammation process and metabolic syndrome [6-25]. NAFLD has been considered as a hepatic manifestation of the metabolic syndrome and is associated with various metabolic abnormalities, including hyperlipidemia, central obesity, and type 2 diabetes [1,26, 27]. So, reduced 
lung function may link to an increased risk of NAFLD. In a previous study, association of reduced lung function with NAFLD was detected among men in a health examination program [28]. However, evidence from large-scale populations about the relationship between reduced lung function and NAFLD is scarce. In addition, it is unclear whether the association can be observed in Chinese population.

For this purpose, the aims of this study were to test the hypotheses that reduced lung function is independently associated with NAFLD in a cross-sectional population study of 1,842 middle-aged and older Chinese subjects.

\section{Methods}

\section{Study population}

In 2011, China launched a national survey of Risk Evaluation of cAncers in Chinese diabeTic Individuals: a lONgitudinal (REACTION) study, which was conducted among 259,657 adults, aged 40 years and older in 25 communities across mainland China, from 2011 to 2012 [29]. The data presented in this article are based on the baseline survey of subsamples from Shanghai in eastern China [30, 31]. All studied individuals came from the Chongming District in Shanghai, China. There were 9930 participants who had complete information about age; sex; smoking and alcohol consumption habits; and a medical history including the use of medications, BMI, and a hepatic ultrasonic examination. Participants meeting the following criteria were excluded: 1) those with a history of known liver diseases such as hepatitis, cirrhosis, or malignancy; 2) those with alcohol consumption greater than $140 \mathrm{~g} / \mathrm{wk}$ for men and $70 \mathrm{~g} / \mathrm{wk}$ for women. Thus, a total of 8850 participants were eventually included in this analysis. Of these, two communities participants received lung function test. 1,842 participants were eventually included in the analysis. The protocol was approved by the Institutional Review Board of Xinhua Hospital affiliated with Shanghai Jiao-Tong University School of Medicine.

\section{Data collection}

A standardized questionnaire was used by trained physicians to collect information such as age; sex; current smoking (yes/no); current drinker (yes/no). Physical activity level was classified as low, moderate, or high according to the International Physical Activity Questionnaire scoring protocol. According to participants' responses to the corresponding questions, family history of diabetes was classified as yes or no.

The details of anthropometric measurements including height, weight, waist circumference, hip circumference were carried by trained physicians. Blood pressure was measured at the right arm with an automated electronic device (OMRON Model1 Plus; Omron Company, Kyoto,
Japan) three times consecutively with 1 min intervals after at least $5 \mathrm{~min}$ rest in the seated position; the three readings were averaged for analysis. Body mass index (BMI) was calculated as weight in kilograms divided by the square of height in meters.

All subjects were assessed after overnight fasting for at least $10 \mathrm{~h}$, Overnight fasting and $2 \mathrm{~h}$ OGTT (Oral Glucose Tolerance Test) 75 g glucose blood samples were collected in tubes containing EDTA and were centrifuged at $4{ }^{\circ} \mathrm{C}$ and stored at $-80{ }^{\circ} \mathrm{C}$ until analysis. The fasting glucose, glucose $2 \mathrm{~h}$ after oral glucose tolerance test, total cholesterol (TC), triglycerides, low-density lipoprotein (LDL) cholesterol and high-density lipoprotein (HDL) cholesterol were measured on an automatic analyzer (Hitachi 7080; Tokyo, Japan). Fasting insulin was determined by RIA (Linco Research, St. Charles, $\mathrm{MO})$. The homeostasis model assessment of insulin resistance (HOMA-IR) was calculated according to the equation described by Matthews et al. [32].

\section{Definition of NAFLD}

Hepatic ultrasonic examination was performed on all participants by two trained ultrasonographists who were blinded to the clinical and laboratory data, using a highresolution B-mode tomographic ultrasound system (Esaote Biomedica $\mathrm{SpA}$, Italy) with a $3.5-\mathrm{MHz}$ probe. Diagnosis of fatty liver by ultrasonography was defined by the presence of at least two of three abnormal

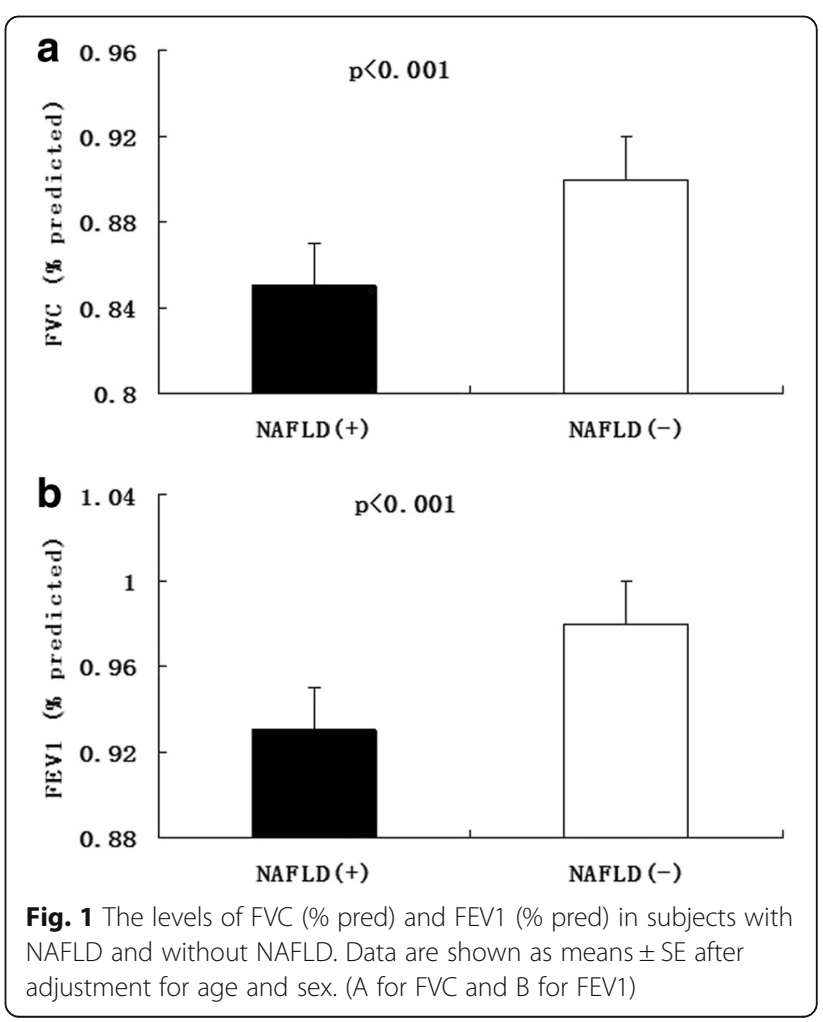


findings: diffusely increased echogenicity of the liver relative to the kidney, ultrasound beam attenuation, and poor visualization of intrahepatic structures. NAFLD was diagnosed by hepatic ultrasound after the exclusion of alcohol abuse and other liver diseases.

\section{Lung function measurements}

Lung function tests including FVC and FEV1 were conducted by a trained physician using Electronic Spirometer (Model BF-II, Jintan, China). Each participant received at least two tests (with acceptable maneuvers) at a seated position and with nose clips in place. The predicted values for FVC and FEV1 were calculated from the following equations obtained in a representative sample of Chinese population [25].

Predicted FVC of $\operatorname{man}=-4.33058-(0.01326 \times$ age [years $])+(0.04669 \times$ height $[\mathrm{cm}])+(0.01664 \times$ weight $[\mathrm{kg}])$.

Predicted FVC of woman $=-4.79287-(0.01326 \times$ age [years $])+(0.04669 \times$ height $[\mathrm{cm}])+(0.01664 \times$ weight $[\mathrm{kg}])$.

Predicted FEV1 of man $=-3.65523-(0.01850 \times$ age [years $])+(0.04283 \times$ height $[\mathrm{cm}])+(0.009228832 \times$ weight $[\mathrm{kg}])$.

Predicted FEV1 of woman $=-4.04947-(0.01850 \times$ age [years $])+(0.04283 \times$ height $[\mathrm{cm}])+(0.009228832 \times$ weight $[\mathrm{kg}])$.

The percentage of predicted values for FEV1, FEV1 (\% pred), equals to FEV1 devided by the predicted values of FEV1. The percentage of predicted values for FVC, FVC (\% pred), equals to $\mathrm{FVC}$ divided by the predicted values of FVC. The ratio of FEV1 to FVC was calculated.

\section{Statistical analysis}

Normally distributed data were expressed as means \pm SD, whereas variables with a skewed distribution were reported as median (interquartile range) and log transformed to approximate normality before analysis. Comparisons of means and proportions were performed with the standard normal $\mathrm{z}$ and $\chi 2$ tests, respectively. Multivariate logistic regression models were used to estimate the odds ratios (ORs) for NAFLD. Potential confounding variables including age, gender, current smoking, family history of diabetes, systolic blood pressure, diastolic blood pressure, fasting plasma glucose, $2 \mathrm{~h}$ OGTT plasma glucose, hemoglobin A1c, waist circumference, BMI, HOMA-IR, TG, TC, LDL-c and HDL-c were controlled in the regression models. All statistical analysis were performed with the SPSS Statistical Package (version 13.0; SPSS Inc., Chicago, IL). $P<0.05$ was considered statistically significant.

\section{Results}

Characteristics of participants according to FVC (\% pred) or FEV1 (\% pred) quartile

Mean values of FVC (\% pred), FEV1 (\% pred) in subjects with NAFLD were significantly lower than in those without
$(0.85 \pm 0.26$ vs. $0.90 \pm 0.28,0.93 \pm 0.29$ vs. $0.98 \pm 0.34$; both $p<0.001$ ) (Fig. 1). Individuals with NAFLD were elder, more likely to be metabolic syndrome, current drinker, and heavy smoking, and had higher levels of BMI, SBP, DBP, waist circumference, hip circumference, waist-hip ratio, fasting plasma glucose FPG, postprandial 2-h plasma glucose, A1C, HOMA-IR, TG, TC, LDL-c, AST, ALT and GGT (all $p$ values $<0.001$ ), and had lower levels of HDL-c $(p<0.001)$ (Table 1).

When analyzed by quartiles of FVC (\% pred) or FEV1 (\% pred) levels, as summarized in Tables 2 and 3, the subjects with lower FVC (\% pred) or FEV1 (\% pred) were more likely to be more metabolic syndrome $(p<0.001)$, more smoker $(p<0.001)$, more drinker $(p<0.001)$, more aged $(p<0.001)$, With respect to metabolic parameters, the subjects in the higher FVC (\% pred) or FEV1 (\% pred) quartiles exhibited low er levels of LDL cholesterol

Table 1 Baseline characteristics of the study participants, grouped according to NAFLD status

\begin{tabular}{|c|c|c|c|}
\hline & $\begin{array}{l}\text { Without NAFLD } \\
(n=1164)\end{array}$ & $\begin{array}{l}\text { With NAFLD } \\
(n=678)\end{array}$ & $P$ value \\
\hline $\begin{array}{l}\text { ATPIII-defined metabolic } \\
\text { syndrome } \mathrm{n}(\%)\end{array}$ & $433(37.21)$ & $526(77.64)$ & $<0.001$ \\
\hline Sex n (\% men) & $376(32.3)$ & 213(31.4) & 0.38 \\
\hline Age $\left(\right.$ years) ${ }^{b}$ & $55.38 \pm 8.10$ & $56.97 \pm 7.55$ & $<0.001$ \\
\hline $\mathrm{BMI}(\mathrm{kg} / \mathrm{m} 2)$ & $23.32 \pm 2.95$ & $26.47 \pm 6.33$ & $<0.001$ \\
\hline Currents smokers n (\%) & 275(23.63) & $178(26.30)$ & $<0.001$ \\
\hline $\mathrm{SBP}(\mathrm{mmHg})$ & $127.49 \pm 20.64$ & $133.10 \pm 20.98$ & $<0.001$ \\
\hline $\mathrm{DBP}(\mathrm{mmHg})$ & $78.99 \pm 10.53$ & $81.77 \pm 10.34$ & $<0.001$ \\
\hline Waist circumference $(\mathrm{cm})$ & $81.18 \pm 10.46$ & $89.42 \pm 8.70$ & $<0.001$ \\
\hline Hip circumference (cm) & $94.12 \pm 6.06$ & $99.05 \pm 6.95$ & $<0.001$ \\
\hline Waist-hip ratio & $0.86 \pm 0.14$ & $0.90 \pm 0.07$ & $<0.001$ \\
\hline $\begin{array}{l}\text { Fasting plasma glucose } \\
(\mathrm{mmol} / \mathrm{l})\end{array}$ & $6.02 \pm 1.45$ & $6.65 \pm 1.93$ & $<0.001$ \\
\hline $2 \mathrm{~h}$ PG (mmol/L) & $7.87 \pm 3.40$ & $9.17 \pm 4.22$ & $<0.001$ \\
\hline $\mathrm{A} 1 \mathrm{C}(\%)$ & $5.82 \pm 0.86$ & $6.25 \pm 1.14$ & $<0.001$ \\
\hline HOMA-IR & $1.47(1.13-1.92)$ & $2.36(1.63-3.27)$ & $<0.001$ \\
\hline Triglycerides (mmol/l) & $1.38 \pm 0.95$ & $2.16 \pm 1.54$ & $<0.001$ \\
\hline HDL-cholesterol (mmol/l) & $1.29 \pm 0.33$ & $1.15 \pm 0.28$ & $<0.001$ \\
\hline LDL-cholesterol (mmol/I) & $2.55 \pm 0.74$ & $2.70 \pm 0.80$ & $<0.001$ \\
\hline AST(units/I) & $15.92 \pm 8.67$ & $22.29 \pm 12.54$ & $<0.001$ \\
\hline ALT(units/I) & $14.15 \pm 10.03$ & $21.19 \pm 15.87$ & $<0.001$ \\
\hline GGT(units/l) & $24.09 \pm 28.30$ & $38.61 \pm 48.45$ & $<0.001$ \\
\hline
\end{tabular}

$S B P$ systolic blood pressure, $D B P$ diastolic blood pressure, $B M I$ body mass index; $2 h P G$ postprandial 2-h plasma glucose, $H b A 1 C$ Glycated hemoglobin, $L D L$ Low-density lipoprotein, $H D L$ high-density lipoprotein, $A L T$ Alanine aminotransferase, AST aspartate aminotransferase, GGT $\gamma$-glutamyltransferase ${ }^{a}$ Data are presented as mean $\pm S D$, median (interquartile range), or number (percent); $P$ value was calculated after adjustment for age, gender

${ }^{\mathrm{b}}$ Not adjusted for itself

'This variables was log transformed before analysis 
Table 2 Characteristic according to quartiles of FVC (\% predicted) ${ }^{a}$

\begin{tabular}{|c|c|c|c|c|c|}
\hline & Quartile $1(n=450)$ & Quartile $2(n=471)$ & Quartile $3(n=460)$ & Quartile $4(n=471)$ & $P$ value \\
\hline FVC (\% predicted) & $0.63 \pm 0.09$ & $0.77 \pm 0.25$ & $0.87 \pm 0.03$ & $1.22 \pm 0.28$ & $<0.001$ \\
\hline FEV1 (\% predicted) & $0.70 \pm 0.18$ & $0.86 \pm 0.15$ & $1.01 \pm 0.26$ & $1.27 \pm 0.31$ & $<0.001$ \\
\hline FEV1/FVC ratio & $0.77 \pm 0.22$ & $0.77 \pm 0.22$ & $0.76 \pm 0.23$ & $0.74 \pm 0.24$ & $<0.001$ \\
\hline MS (n, \%) & 294(65.33) & $271(57.53)$ & $253(55.00)$ & $232(49.26)$ & $<0.001$ \\
\hline NAFLD (n, \%) & 193(42.89) & 188(39.92) & $160(34.78)$ & 137(29.09) & $<0.001$ \\
\hline Age (years) ${ }^{b}$ & $56.64 \pm 8.23$ & $55.65 \pm 7.87$ & $55.37 \pm 7.48$ & $56.31 \pm 7.91$ & 0.007 \\
\hline Male (n, \%) & $45(10.00)$ & $78(16.56)$ & 168(36.52) & 384(81.53) & $<0.001$ \\
\hline Current smoking (n, \%) & $51(11.33)$ & $66(14.01)$ & $87(18.91)$ & $166(35.24)$ & $<0.001$ \\
\hline Current drinker (n, \%) & $61(13.56)$ & 88(18.68) & $86(18.70)$ & 183(38.85) & $<0.001$ \\
\hline Regular exerciser $(n, \%)$ & $265(58.89)$ & $282(59.87)$ & $275(59.78)$ & $284(60.29)$ & 0.978 \\
\hline $\mathrm{BMI}\left(\mathrm{kg} / \mathrm{m}^{2}\right)$ & $24.82 \pm 3.97$ & $25.09 \pm 3.59$ & $24.48 \pm 3.27$ & $24.73 \pm 3.34$ & 0.102 \\
\hline $\mathrm{SBP}(\mathrm{mmHg})$ & $137.56 \pm 19.42$ & $134.32 \pm 18.82$ & $131.51 \pm 19.15$ & $135.78 \pm 18.95$ & $<0.001$ \\
\hline $\mathrm{DBP}(\mathrm{mmHg})$ & $82.40 \pm 10.44$ & $82.16 \pm 10.87$ & $80.98 \pm 9.98$ & $82.53 \pm 10.57$ & $<0.001$ \\
\hline WC (cm) & $86.54 \pm 11.48$ & $85.76 \pm 11.11$ & $83.93 \pm 10.31$ & $84.73 \pm 10.45$ & 0.002 \\
\hline FPG (mmol/L) & $6.54 \pm 1.87$ & $6.65 \pm 2.21$ & $6.32 \pm 1.75$ & $6.31 \pm 1.36$ & 0.011 \\
\hline $2 \mathrm{~h} \mathrm{PG}(\mathrm{mmol} / \mathrm{L})$ & $9.42 \pm 4.23$ & $9.43 \pm 4.57$ & $8.70 \pm 4.01$ & $8.38 \pm 3.61$ & $<0.001$ \\
\hline $\mathrm{A} 1 \mathrm{C}(\%)$ & $5.90 \pm 1.10$ & $5.94 \pm 1.23$ & $5.75 \pm 1.01$ & $5.67 \pm 0.83$ & $<0.001$ \\
\hline HOMA-IRC & $2.32(1.44-3.31)$ & $1.84(1.30-2.68)$ & $1.75(1.22-2.50)$ & $1.66(1.18-2.32)$ & 0.029 \\
\hline eGFR & $124.75 \pm 25.25$ & $122.57 \pm 21.90$ & $120.30 \pm 19.95$ & $114.73 \pm 21.85$ & $<0.001$ \\
\hline Triglycerides $(\mathrm{mmol} / \mathrm{L})^{c}$ & $1.88 \pm 1.27$ & $1.76 \pm 1.30$ & $1.63 \pm 1.05$ & $1.89 \pm 1.65$ & 0.009 \\
\hline TC $(\mathrm{mmol} / \mathrm{L})$ & $5.14 \pm 0.96$ & $5.07 \pm 0.88$ & $4.87 \pm 0.85$ & $4.86 \pm 0.88$ & $<0.001$ \\
\hline $\mathrm{HDL}-\mathrm{c}(\mathrm{mmol} / \mathrm{L})$ & $1.34 \pm 0.32$ & $1.36 \pm 0.31$ & $1.31 \pm 0.32$ & $1.29 \pm 0.33$ & 0.004 \\
\hline LDL-c (mmol/L) & $2.84 \pm 0.75$ & $2.81 \pm 0.74$ & $2.73 \pm 0.70$ & $2.71 \pm 0.72$ & $<0.001$ \\
\hline ALT (units/l) & $21.37 \pm 17.36$ & $21.08 \pm 15.98$ & $19.78 \pm 14.06$ & $17.82 \pm 11.15$ & $<0.001$ \\
\hline AST (units/l) & $25.35 \pm 13.05$ & $24.28 \pm 12.53$ & $24.13 \pm 10.75$ & $22.14 \pm 7.31$ & $<0.001$ \\
\hline GGT (units/l) & $42.19 \pm 42.29$ & $36.22 \pm 51.10$ & $30.96 \pm 33.42$ & $29.91 \pm 37.36$ & $<0.001$ \\
\hline
\end{tabular}

SBP systolic blood pressure, DBP diastolic blood pressure, BMI body mass index, OGTT Oral Glucose Tolerance Test, FPG Fasting Plasma Glucose, 2 h PG postprandial 2-h Plasma Glucose, HbA1C Glycated hemoglobin, LDL Low-density lipoprotein, HDL high-density lipoprotein, ALT Alanine aminotransferase, AST aspartate aminotransferase, GGT $\gamma$-glutamyltransferase

${ }^{a}$ Data are presented as mean $\pm S D$, median (interquartile range), or number (percent); $P$ value was calculated after adjustment for age, gender

${ }^{b}$ Not adjusted for itself

'This variables was log transformed before analysis

$(p<0.001)$. However, elevated FVC (\% pred) or FEV1 (\% pred) levels showed no association with the regular exerciser $(p>0.05)$.

\section{Association between quartiles of FVC (\% pred) and FEV1 (\% pred) and NAFLD}

As shown in Table 4, the lowest quartile of FVC (\% pred) and FEV1 (\% pred) was associated with increased odds of NAFLD, with age- and sex-adjusted odds ratio (OR) of 1.82 and 1.74, respectively (95\% confidential interval (CI), $1.38-2.39$ and $95 \%$ CI, 1.32-2.28; both $p<0.001$ ). Further adjustments for current smoking, family history of diabetes systolic blood pressure, diastolic blood pressure, fasting plasma glucose, 2 h OGTT plasma glucose, Hemoglobin A1c, waist circumference, BMI, HOMA-IR, TG, TC, LDL-c and HDL-c did not eliminate the associations (OR, 1.37, 95\% CI, 1.18-1.97, $p<0.001$ and OR,1.24, 95\% CI, 1.11-1.87, $p=0.009$ ).

\section{Discussion}

In the present study, we found that impaired lung function as measured by FVC and FEV1 was significantly and inversely associated with prevalence of NAFLD in a middle-aged and elderly population without chronic pulmonary diseases after adjustment for a wide range of variables including age, gender, current smoking, family history of diabetes, systolic blood pressure, diastolic blood pressure, fasting plasma glucose, $2 \mathrm{~h}$ OGTT plasma glucose, hemoglobin A1c, waist circumference, BMI, HOMA-IR and lipid profile.

Our results are consistent with previous study that restrictive lung function (reduced FVC and FEV1) but not 
Table 3 Characteristic according to quartiles of FEV1 (\% predicted) ${ }^{a}$

\begin{tabular}{|c|c|c|c|c|c|}
\hline & Quartile $1(n=454)$ & Quartile $2(n=467)$ & Quartile $3(n=464)$ & Quartile $4(n=467)$ & $P$ value \\
\hline FEV1 (\%) & $0.66 \pm 0.11$ & $0.83 \pm 0.03$ & $0.95 \pm 0.05$ & $1.42 \pm 0.26$ & $<0.001$ \\
\hline FVC (\%) & $0.66 \pm 0.14$ & $0.79 \pm 0.12$ & $0.92 \pm 0.20$ & $1.13 \pm 0.30$ & $<0.001$ \\
\hline FEV1/FVC ratio & $73.21 \pm 22.91$ & $77.23 \pm 22.01$ & $76.63 \pm 22.81$ & $78.02 \pm 22.61$ & $<0.001$ \\
\hline MS (n, \%) & $282(62.11)$ & $273(58.46)$ & $255(54.96)$ & 240(51.39) & $<0.001$ \\
\hline NAFLD (n, \%) & $200(44.05)$ & $171(36.62)$ & 162(34.91) & 145(30.79) & $<0.001$ \\
\hline Age $(\text { years })^{b}$ & $56.37 \pm 8.22$ & $55.51 \pm 7.72$ & $55.17 \pm 8.12$ & $56.68 \pm 7.50$ & 0.004 \\
\hline Male $(n, \%)$ & $47(10.35)$ & $82(17.56)$ & $164(35.34)$ & $382(81.80)$ & $<0.001$ \\
\hline Current smoking $(\mathrm{n}, \%)$ & $55(12.11)$ & $68(14.56)$ & $87(18.75)$ & 159(34.05) & $<0.001$ \\
\hline Current drinker $(n, \%)$ & $64(14.10)$ & $90(19.27)$ & $89(19.18)$ & 175(37.47) & $<0.001$ \\
\hline Regular exerciser (n, \%) & $263(57.93)$ & $285(61.03)$ & $272(58.62)$ & $287(61.46)$ & 0.872 \\
\hline $\mathrm{BMI}\left(\mathrm{kg} / \mathrm{m}^{2}\right)$ & $24.81 \pm 3.93$ & $24.76 \pm 3.64$ & $25.05 \pm 3.42$ & $24.74 \pm 3.32$ & 0.556 \\
\hline $\mathrm{SBP}(\mathrm{mmHg})$ & $136.28 \pm 19.26$ & $135.84 \pm 19.25$ & $134.38 \pm 18.12$ & $133.54 \pm 20.55$ & 0.002 \\
\hline $\mathrm{DBP}(\mathrm{mmHg})$ & $82.50 \pm 10.43$ & $82.12 \pm 10.49$ & $82.06 \pm 10.36$ & $81.90 \pm 10.81$ & $<0.001$ \\
\hline WC $(\mathrm{cm})$ & $86.04 \pm 11.68$ & $85.06 \pm 11.34$ & $86.05 \pm 10.27$ & $84.70 \pm 10.29$ & 0.015 \\
\hline FPG (mmol/L) & $6.55 \pm 1.88$ & $6.50 \pm 3.64$ & $6.48 \pm 1.90$ & $6.36 \pm 1.49$ & 0.004 \\
\hline $2 \mathrm{~h} \mathrm{PG}(\mathrm{mmol} / \mathrm{L})$ & $9.30 \pm 4.20$ & $9.10 \pm 4.39$ & $9.03 \pm 4.09$ & $8.47 \pm 3.79$ & 0.017 \\
\hline$A 1 C(\%)$ & $5.88 \pm 1.07$ & $5.88 \pm 1.14$ & $5.83 \pm 1.11$ & $5.70 \pm 0.93$ & 0.035 \\
\hline HOMA-IR & $2.30(1.42-3.33)$ & $1.79(1.28-2.63)$ & $1.70(1.23-2.52)$ & $1.64(1.15-2.31)$ & 0.025 \\
\hline eGFR & $124.85 \pm 25.05$ & $121.73 \pm 21.24$ & $119.85 \pm 21.90$ & $115.14 \pm 21.27$ & $<0.001$ \\
\hline Triglycerides (mmol/L) & $1.86 \pm 1.26$ & $1.73 \pm 1.35$ & $1.80 \pm 1.38$ & $1.77 \pm 1.42$ & 0.035 \\
\hline $\mathrm{TC}(\mathrm{mmol} / \mathrm{L})$ & $5.06 \pm 0.93$ & $4.95 \pm 1.02$ & $4.98 \pm 0.84$ & $4.92 \pm 0.82$ & 0.125 \\
\hline $\mathrm{HDL}-\mathrm{c}(\mathrm{mmol} / \mathrm{L})$ & $1.33 \pm 0.32$ & $1.32 \pm 0.30$ & $1.32 \pm 0.31$ & $1.32 \pm 0.31$ & 0.031 \\
\hline LDL-c (mmol/L) & $2.83 \pm 0.75$ & $2.79 \pm 0.79$ & $2.70 \pm 0.67$ & $2.69 \pm 0.68$ & 0.007 \\
\hline ALT(units/l) & $21.22 \pm 14.07$ & $21.15 \pm 14.28$ & $19.94 \pm 13.53$ & $18.17 \pm 13.09$ & $<0.001$ \\
\hline AST(units/l) & $24.97 \pm 12.85$ & $24.31 \pm 12.66$ & $24.03 \pm 11.49$ & $22.47 \pm 10.29$ & $<0.001$ \\
\hline GGT(units/l) & $41.25 \pm 37.73$ & $38.07 \pm 43.25$ & $31.24 \pm 34.71$ & $28.99 \pm 36.52$ & $<0.001$ \\
\hline
\end{tabular}

SBP systolic blood pressure, DBP diastolic blood pressure, BMl body mass index, OGTT Oral Glucose Tolerance Test, $F P G$ Fasting Plasma Glucose, $2 \mathrm{~h} P G$ postprandial 2-h Plasma Glucose, HbA1C HbA1C hemoglobin A1C, eGFR estimate the glomerular filtration rate, LDL Low-density lipoprotein, HDL high-density lipoprotein, ALT Alanine aminotransferase, AST aspartate aminotransferase, GGT $\gamma$-glutamyltransferase

${ }^{a}$ Data are presented as mean $\pm S D$, median (interquartile range), or number (percent); $P$ value was calculated after adjustment for age, gender

${ }^{\mathrm{b}}$ Not adjusted for itself

'This variables was log transformed before analysis

Table 4 Odds ratio for the non-alcoholic fatty liver disease according to quartiles of FVC (\% pred) or FEV1 (\% pred)

\begin{tabular}{|c|c|c|c|c|c|}
\hline & \multicolumn{5}{|l|}{ FVC(\% pred) } \\
\hline & Quartile 1 & Quartile 2 & Quartile 3 & Quartile 4 & $P$ value \\
\hline Model 1 & $1.82(1.38-2.39)$ & $1.60(1.23-2.12)$ & $1.29(0.97-1.70)$ & 1 & $<0.001$ \\
\hline Model 2 & $1.65(1.27-2.24)$ & $1.39(1.18-2.01)$ & $1.12(0.87-1.52)$ & 1 & $<0.001$ \\
\hline \multirow[t]{3}{*}{ Model 3} & $1.37(1.18-1.97)$ & $1.19(1.08-1.82)$ & $1.04(0.79-1.28)$ & 1 & $<0.001$ \\
\hline & \multicolumn{5}{|l|}{ FEV1(\% pred) } \\
\hline & Quartile 1 & Quartile 2 & Quartile 3 & Quartile 4 & $P$ value \\
\hline Model 1 & $1.74(1.32-2.28)$ & $1.27(0.96-1.67)$ & $1.18(0.90-1.55)$ & 1 & $<0.001$ \\
\hline Model 2 & $1.48(1.24-2.12)$ & $1.13(0.89-1.48)$ & $1.09(0.81-1.39)$ & 1 & 0.004 \\
\hline Model 3 & $1.24(1.11-1.87)$ & $1.07(0.76-1.21)$ & $1.03(0.72-1.19)$ & 1 & 0.009 \\
\hline
\end{tabular}

Model 1, adjusted for age and sex; model 2, further adjusted for current smoking, family history of diabetes systolic blood pressure and diastolic blood pressure; model 3, further adjusted for fasting plasma glucose, 2 h OGTT plasma glucose, Hemoglobin A1c, waist circumference, BMI, HOMA-IR, TG, TC, LDL-c and HDL-c 
obstructive pulmonary function (FEV1-to-FVC ratio) is associated with the development of NAFLD [28]. The underlying mechanisms relating reduced lung function to this type of metabolic disorder remain unclear; integration of inflammatory process and metabolic pathways in NAFLD patients may be a pivotal underlying mechanism link between reduced pulmonary function and incident NAFLD. Previous studies have demonstrated that a strong association between both restrictive and obstructive lung patterns and inflammatory markers [33, 34]. As we best known, low-grade systemic inflammation play a causal role in the development of NAFLD. Thus, the inflammatory process may contribute to the association between reduced lung function and NAFLD. However, the measurement of inflammatory markers was absent, limiting the ability to access the role of this factor in the association in the present study.

In our study, we observed that the positive association of FVC (\% pred) in particular and FEV1 (\% pred) with metabolic abnormalities and components of the insulin resistance syndrome, which is consistent with several previous studies that have reported associations between restrictive lung patterns with glucose metabolism and metabolic syndrome [6-23]. Moreover, our study also demonstrated that FVC (\% pred) and FEV1 (\% pred) were associated with insulin sensitivity as measured by the HOMA-IR. It has been well demonstrated that insulin resistance plays a key role in the development of NAFLD $[35,36]$. Metabolic risk factors closely associated with insulin resistance (BMI, glucose, waist circumference, blood pressure, triglycerides, and HDL cholesterol) may affect the association of FVC (\% pred), FEV1 (\% pred) and NAFLD. However, our further analysis indicated that the effects of reduced lung function on NAFLD were independent of metabolic syndrome features.

The strengths of this study include the communitybased sample, standardized spirometric techniques, extensive data on potential confounders, and a large sample size that increased precision and permitted multiple statistical adjustments. However, several limitations of our study have to be addressed. First, due to the cross-sectional nature of the current study, no causal inference can be drawn. Prospective studies are needed to clarify their precise interrelationship. Also, it has yet to be seen whether our results in middle-aged and older Chinese subjects can be generalized to younger populations or other ethnic groups. Secondly, liver biopsies, the best diagnostic tool for confirming NAFLD, were not available in our participants. The diagnosis of NAFLD was based on ultrasonic examination, which is not sensitive enough to detect mild steatosis. However, this method is the most widely used noninvasive technique to detect fatty infiltration of the liver in clinical practice and epidemiological studies, and it has been reported to have a sensitivity of $89 \%$ and specificity of $93 \%$ for the identification of fatty liver [37]. Third, the lack of inflammatory markers, which precluded more detailed investigations of the causal pathway. Furthermore, sleep duration/quality and symptoms of sleep apnea which was related to both insulin resistance and lung function may affect the association of impaired lung function and NAFLD, however, due to study design defect, we couldn't further analyze the effect in this study.

\section{Conclusions}

We have found that impaired lung function was associated with NAFLD in middle-aged and elderly Chinese population. These findings suggest the need to screen impaired of lung function in people without respiratory disease for the presence of NAFLD.

\section{Abbreviations}

ALT: Alanine aminotransferase; AST: Aspartate aminotransferase; BMI: Body mass index; FEV1: Forced expiratory volume in one second; FVC: Forced vital capacity; GGT: y-glutamyltransferase; HDL: High-density lipoprotein cholesterol; HOMA-IR: Homeostasis model assessment of insulin resistance; LDL: Low-density lipoprotein cholesterol; NAFLD: Nonalcoholic fatty liver disease; Ors: Odds ratios:- TC: Total cholesterol

\section{Acknowledgements}

Not applicable.

\section{Funding}

This work was supported by National Natural Science Foundation of China (81370953, 81300667, 81370935), Shanghai Health System Outstanding Young Talents Training Program (XYQ2013098), Shanghai Science and Technology Commission (15411953200, 10411956600, 14ZR1427400)

Shanghai Education Committee Key Program (14zz110), National Clinical Research Center for Metabolic Diseases (2013BAI09B13), National Key New Drug Creation and Manufacturing Program of Ministry of Science and Technology (2012ZX09303006-001) and State Key Development Program for Basic Research of China (2012CB517501). We thank the field workers for their contribution and the participants for their cooperation.

\section{Availability of data and materials}

The data that support the findings of this study are available from REACTION Study Group but restrictions apply to the availability of these data, which were used under license for the current study, and so are not publicly available. Data are however available from the authors upon reasonable request and with permission of REACTION Study Group.

\section{Authors' contributions}

Conceived and designed the experiments: $Z Y$ and QS. Analyzed the data: $L Q$, WWZ, ZY, YXN, SL, YX, NL, HMZ, QS. Contributed reagents/materials/analysis tools: LQ, WWZ, ZY, GN, JGF,QS. Wrote the paper: LQ, WWZ, ZY, QS. All authors read and approved the final manuscript.

Competing interest

The authors declare that they have no competing interests.

Consent for publication

Not applicable.

Ethics approval and consent to participate

The protocol was approved by the Ethics Committee of Xinhua Hospital Affiliated to Shanghai Jiaotong University School of Medicine. Written consent was obtained from all the participants. The methods were carried out in accordance with the approved guidelines. 


\section{Publisher's Note}

Springer Nature remains neutral with regard to jurisdictional claims in published maps and institutional affiliations.

\section{Author details \\ 'Department of Endocrinology, Xinhua Hospital Chongming Branch, Shanghai Jiao Tong University School of Medicine, Shanghai, China. ${ }^{2}$ Department of Endocrinology, Xinhua Hospital, Shanghai Jiao Tong University School of Medicine, 1665 Kongjiang Road, Shanghai, China. ${ }^{3}$ Department of Endocrinology and Metabolism, Key Laboratory for Endocrine and Metabolic Diseases of Ministry of Health, Ruilin Hospital, Shanghai Jiao Tong University School of Medicine, E-Institute of Shanghai Universities, Shanghai, China. ${ }^{4}$ Department of Gastroenterology, Shanghai Key Laboratory of Children's Digestion and Nutrition, Xinhua Hospital, Shanghai Jiaotong University School of Medicine, Shanghai, China.}

\section{Received: 8 October 2016 Accepted: 11 March 2017}

\section{Published online: 22 March 2017}

\section{References}

1. Fan JG, Farrell GC. Epidemiology of non-alcoholic fatty liver disease in China. J Hepatol. 2009;50:204-10.

2. Gaggini M, Morelli M, Buzzigoli E, DeFronzo RA, Bugianesi E, Gastaldelli A. Non-alcoholic fatty liver disease (NAFLD) and its connection with insulin resistance, dyslipidemia, atherosclerosis and coronary heart disease. Nutrients. 2013:5:1544-60.

3. Anstee QM, McPherson S, Day CP. How big a problem is non-alcoholic fatty liver disease? BMJ. 2011;343:d3897.

4. Williamson RM, Price JF, Glancy S, Perry E, Nee LD, Hayes PC, et al. Prevalence of and risk factors for hepatic steatosis and nonalcoholic Fatty liver disease in people with type 2 diabetes: the Edinburgh Type 2 Diabetes Study. Diabetes Care. 2011;34:1139-44.

5. Leung TM, Nieto N. CYP2E1 and oxidant stress in alcoholic and non-alcoholic fatty liver disease. J Hepatol. 2013;58:395-8.

6. Hickson DA, Burchfiel CM, Liu J, Petrini MF, Harrison K, White WB, et al. Diabetes, impaired glucose tolerance, and metabolic biomarkers in individuals with normal glucose tolerance are inversely associated with lung function: the Jackson Heart Study. Lung. 2011;189:311-21.

7. Ubhi BK, Riley JH, Shaw PA, Lomas DA, Tal-Singer R, MacNee W, et al. Metabolic profiling detects biomarkers of protein degradation in COPD patients. Eur Respir J. 2012:40:345-55.

8. Walter RE, Beiser A, Givelber RJ, O'Connor GT, Gottlieb DJ. Association between glycemic state and lung function: the Framingham Heart Study. Am J Respir Crit Care Med. 2003:167:911-6.

9. McKeever TM, Weston PJ, Hubbard R, Fogarty A. Lung function and glucose metabolism: an analysis of data from the Third National Health and Nutrition Examination Survey. Am J Epidemiol. 2005;161:546-56.

10. Davis WA, Knuiman M, Kendall P, Grange V, Davis TM. Fremantle Diabetes Study. Glycemic exposure is associated with reduced pulmonary function in type 2 diabetes: the Fremantle Diabetes Study. Diabetes Care. 2004;27:752-7.

11. Nakajima K, Ebata M, Saito M. The relationship between low vital capacity and impaired glucose metabolism in men. Diabet Med. 2010;27:1460-1.

12. Klein OL, Krishnan JA, Glick S, Smith LJ. Systematic review of the association between lung function and Type 2 diabetes mellitus. Diabet Med. 2010;27:977-87.

13. van den Borst B, Gosker HR, Zeegers MP, Schols AM. Pulmonary function in diabetes: a meta-analysis. Chest. 2010;138:393-406.

14. Heianza Y, Arase Y, Tsuji H, Saito K, Amakawa K, Hsieh SD, et al. Low lung function and risk of type 2 diabetes in Japanese men: the Toranomon Hospital Health Management Center Study 9 (TOPICS 9). Mayo Clin Proc. 2012;87:853-61.

15. Kwon CH, Rhee EJ, Song JU, Kim JT, Kwag HJ, Sung KC. Reduced lung function is independently associated with increased risk of type 2 diabetes in Korean men. Cardiovasc Diabetol. 2012;11:38.

16. Engstrom $\mathrm{G}$, Janzon L. Risk of developing diabetes is inversely related to lung function: a population-based cohort study. Diabet Med. 2002;19:167-70.

17. Ford ES, Mannino DM. National Health and Nutrition Examination Survey Epidemiologic Follow-up Study. Prospective association between lung function and the incidence of diabetes: findings from the National Health and Nutrition Examination Survey Epidemiologic Follow-up Study. Diabetes Care. 2004;27:2966-70
18. Rana JS, Mittleman MA, Sheikh J, Hu FB, Manson JE, Colditz GA, et al. Chronic obstructive pulmonary disease, asthma, and risk of type 2 diabetes in women. Diabetes Care. 2004;27:2478-84.

19. Yeh HC, Punjabi NM, Wang NY, Pankow JS, Duncan BB, Brancati FL. Vital capacity as a predictor of incident type 2 diabetes: the Atherosclerosis Risk in Communities study. Diabetes Care. 2005;28:1472-9.

20. Song Y, Klevak A, Manson JE, Buring JE, Liu S. Asthma, chronic obstructive pulmonary disease, and type 2 diabetes in the Women's Health Study. Diabetes Res Clin Pract. 2010;90:365-71.

21. Yeh HC, Punjabi NM, Wang NY, Pankow JS, Duncan BB, Cox CE, et al. Cross-sectional and prospective study of lung function in adults with type 2 diabetes: the Atherosclerosis Risk in Communities (ARIC) study. Diabetes Care. 2008:31:741-6.

22. Wannamethee SG, Shaper AG, Rumley A, Sattar N, Whincup PH, Thomas MC, et al. Lung function and risk of type 2 diabetes and fatal and nonfatal major coronary heart disease events: possible associations with inflammation. Diabetes Care. 2010;33:1990-6.

23. Engstrom G, Hedblad B, Nilsson P, Wollmer P, Berglund G, Janzon L. Lung function, insulin resistance and incidence of cardiovascular disease: a longitudinal cohort study. J Intern Med. 2003;253:574-81.

24. Engström G, Lind $P$, Hedblad B, Wollmer P, Stavenow L, Janzon L, et al. Lung function and cardiovascular risk: relationship with inflammationsensitive plasma proteins. Circulation. 2002;106:2555-60.

25. Ma Z, Liu $Y, X u Y$, , Huang $Y, X u M$, Zhu $X$, et al. Impaired lung function is associated with increased carotid intima-media thickness in middle-aged and elderly Chinese. PLoS One. 2013:8:e53153.

26. Marchesini G, Bugianesi E, Forlani G, Cerrelli F, Lenzi M, Manini R, et al. Nonalcoholic fatty liver, steatohepatitis, and the metabolic syndrome. Hepatology. 2003;37:917-23

27. Marchesini G, Brizi M, Bianchi G, Tomassetti S, Bugianesi E, Lenzi M, et al. Nonalcoholic fatty liver disease: a feature of the metabolic syndrome. Diabetes. 2001;50:1844-50.

28. Jung DH, Shim JY, Lee HR, Moon BS, Park BJ, Lee YJ. Relationship between non-alcoholic fatty liver disease and pulmonary function. Intern Med J. 2012:42(5):541-6.

29. Ning G, Reaction Study Group. Risk Evaluation of cAncers in Chinese diabeTic Individuals: a IONgitudinal (REACTION) study. J Diabetes. 2012:4:172-3

30. Yang Z, Zhang H, Niu Y, Zhang W, Zhu L, Li X, et al. Circulating periostin in relation to insulin resistance and nonalcoholic fatty liver disease among overweight and obese subjects. Sci Rep. 2016;6:37886.

31. Yang Z, Yan C, Liu G, Niu Y, Zhang W, Lu S, et al. Plasma selenium levels and nonalcoholic fatty liver disease in Chinese adults: a cross-sectional analysis. Sci Rep. 2016:6:37288.

32. Matthews DR, Hosker JP, Rudenski AS, Naylor BA, Treacher DF, Turner RC. Homeostasis model assessment: insulin resistance and beta-cell function from fasting plasma glucose and insulin concentrations in man. Diabetologia. 1985;28(7):412-9.

33. Gan WQ, Man SF, Senthilselvan A, Sin DD. Association between chronic obstructive pulmonary disease and systemic inflammation: a systematic review and a meta-analysis. Thorax. 2004;59:574-80.

34. Qin L, Yang Z, Zhang W, Gu H, Lu S, Shi Q, et al. Association between metabolic syndrome and lung function in middle-aged and elderly Chinese individuals. Diabetes Res Clin Pract. 2015;108:e46-8.

35. Gariani K, Philippe J, Jornayvaz FR. Non-alcoholic fatty liver disease and insulin resistance: from bench to bedside. Diabetes Metab. 2013;39:16-26.

36. Takamura T, Misu H, Ota T, Kaneko S. Fatty liver as a consequence and cause of insulin resistance: lessons from type 2 diabetic liver. Endocr J. 2012;59:745-63.

37. Joseph AE, Saverymuttu SH, Al-Sam S, Cook MG, Maxwell JD. Comparison of liver histology with ultrasonography in assessing diffuse parenchymal liver disease. Clin Radiol. 1991:43:26-31. 